\title{
Nutrição mineral da fava d'anta
}

\author{
Candido Alves da Costa; Daniel Soares Alves; Luiz Arnaldo Fernandes; Ernane Ronie Martins; Iure \\ Giuliano Braga Souza; Regynaldo Arruda Sampaio; Paulo Sérgio do Nascimento Lopes
}

UFMG, ICA, C. Postal 135, 39404-006 Montes Claros-MG; E-mail: candido-costa@nca.ufmg.br.

\begin{abstract}
RESUMO
Com o objetivo de caracterizar as limitações nutricionais na produção, no crescimento, nutrição e teores de flavonóides totais em fava-d'anta, um experimento foi conduzido em casa de vegetação com amostras de 0-20 cm de profundidade de um Latossolo Vermelho sob vegetação de cerrado. O delineamento experimental utilizado foi o inteiramente casualizado com 13 tratamentos e três repetições. Os tratamentos constaram da adubação com elemento faltante (omitindo-se a calagem e cada um dos nutrientes $\mathrm{N}, \mathrm{P}, \mathrm{K}, \mathrm{S}, \mathrm{B}, \mathrm{Cu}$, $\mathrm{Fe}$ e $\mathrm{Zn}$ ). Verificou-se que a fava d'anta é tolerante à acidez do solo e sensível à deficiência de fósforo e potássio. $\mathrm{O}$ teor de flavonóides totais não variou entre os tratamentos.
\end{abstract}

Palavras-chave: Dimorphandra mollis, plantas medicinais, deficiência mineral.

\begin{abstract}
Mineral Nutrition of Dimorphandra mollis

Aiming to characterize the nutritional limitations on growth, nutrition and total flavonoids concentration of the Dimorphandra mollis, an experiment was conducted in a greenhouse with $0-20 \mathrm{~cm}$ samples of an Oxisol under Cerrado vegetation. The experimental design was completely randomized with 13 treatments and three replications. The treatments consisted of fertilization with missing element technique (with omission of liming and each one of the macro and micronutrients $\mathrm{N}, \mathrm{P}, \mathrm{K}, \mathrm{S}, \mathrm{B}, \mathrm{Cu}, \mathrm{Fe}$ and $\mathrm{Zn}$ ). Dimorphandra mollis plant is tolerant to acid soil and responsive to the omission of phosphorus and potassium. The total flavonoids concentration was not influenced by the treatments.
\end{abstract}

Keywords: Dimorphandra mollis, medicinal plants, nutrient deficiency.

\section{(Recebido para publicação em 30 de novembro de 2005; aceito em 27 de fevereiro de 2007)}

\begin{abstract}
A Fava-d'anta (Dimorphandra mollis Benth), espécie arbórea, com 8-14 metros de altura é encontrada em diversas regiões do país. A planta cresce no Cerrado dos estados do Amazonas, Bahia, Goiás, Maranhão, Mato Grosso do Sul, Minas Gerais, Pará, Piauí, São Paulo, Tocantins e Distrito Federal (Almeida, 1998). A espécie é adaptada às condições de baixa precipitação pluviométrica, com floração e enfolhamento no período úmido e frutificação e queda de folhas na época seca do ano (Lorenzi, 2002).

Os frutos são vagens semideiscentes, achatadas, de coloração marrom, medindo até $15 \mathrm{~cm}$ de comprimento, com mesocarpo farináceo adocicado, porém de sabor desagradável, matéria prima extrativa de rutina e outros flavonóides glicosilados destinados à indústria farmacêutica (Ferreira et al., 2001; Lorenzi, 2002). Possui atividade vitamínica P, própria dos bioflavonóides e capacidade de normalizar a resistência e permeabilidade dos capilares sanguíneos, especialmente quando associada à vitamina $\mathrm{C}$ (Ferreira et al., 2001). A produção de rutina atinge no Brasil cerca de 100 toneladas anuais e a maior parte é destinada à exportação (Ribeiro et al., 2005).
\end{abstract}

Há grande potencial para exploração de gomas das sementes pela indústria de alimentos, melhorando as propriedades dos produtos, principalmente pelo efeito espessante, estabilizante e geleificante por causa do teor de galactomanano (Panegassi et al., 2000).

$\mathrm{O}$ estresse nutricional decorrente da deficiência ou do excesso de nutrientes que interferem no metabolismo vegetal é importante fator na redução da produtividade, notadamente em solos tropicais com baixa disponibilidade de nutrientes e elevada acidez. A busca por plantas adaptadas a estas condições tem sido justificada pela ampla variabilidade inter e intra-específica quanto à maioria dos estresses nutricionais que interferem nos processos de absorção, transporte e utilização de nutrientes pelas plantas (Dechen et al., 1999).

Sabe-se que as plantas adaptadas aos solos minerais ácidos têm mecanismos variáveis visando os fatores químicos adversos destes ambientes. Estes mecanismos são interrelacionados e determinam as exigências por fertilizantes pelas plantas (Matos et al., 1999).

As exigências nutricionais das espécies nativas têm sido bastante diferen- ciadas. Braga et al. (1995), utilizando a técnica do elemento faltante, em solo de baixa fertilidade, verificaram que a quaresmeira (Tibouchina granulosa) mostrou alta exigência nutricional, respondendo à adubação com todos os macro e micronutrientes, enquanto, a Acacia mangium respondeu apenas ao $\mathrm{P}, \mathrm{N}$ e $\mathrm{S}$, e a pereira (Platicyamus regnellii) ao $\mathrm{N}, \mathrm{P}, \mathrm{Ca}$ e $\mathrm{S}$.

Utilizando essa mesma técnica no crescimento em altura do cedro (Cedrela fissillis), do jacaré (Piptadenia gonoacantha), do pau-ferro (Caesalpinea ferrea) e da canafístula (Senna multijuga), o P, S e N foram altamente limitantes. Contudo, a resposta a $\mathrm{Ca}, \mathrm{Mg}$ e micronutrientes foi diferenciada entre as espécies florestais. Os micronutrientes limitaram o crescimento da canafístula e do pau-ferro. O K não foi limitante em nenhuma das espécies, evidenciando baixa exigência deste nutriente (Renó et al., 1993).

Estudando o desenvolvimento inicial da aroeira em solos de cerrado, Melo et al. (1981) verificaram que a obtenção de mudas de boa qualidade somente é possível se as limitações químicas do solo forem corrigidas. 
O presente trabalho teve como objetivo caracterizar as limitações nutricionais no crescimento, nutrição e teor de flavonóides totais em plantas de fava-d'anta, por meio da técnica do elemento faltante.

\section{MATERIAL E MÉTODOS}

O experimento foi conduzido em casa de vegetação, EM Montes Claros, na Universidade Federal de Minas Gerais.

Foram selecionadas 300 sementes de fava d'anta (Dimorphandra mollis Benth) coletadas em áreas de Cerrado na região norte do estado de Minas Gerais.

As sementes foram submetidas a tratamento com ácido sulfúrico concentrado durante uma hora objetivando a quebra da dormência, sendo lavadas posteriormente em água corrente durante 20 minutos. A semeadura foi realizada em bandejas plásticas de 30 x 50 x $15 \mathrm{~cm}$, inicialmente desinfestadas com água sanitária, contendo a camada de $15 \mathrm{~cm}$ de areia lavada autoclavada por $20 \mathrm{mi}$ nutos à pressão de 1 atm e umedecida com água destilada. Após a semeadura, as bandejas foram cobertas com filme plástico permanecendo em $\mathrm{BOD}$ a $28^{\circ} \mathrm{C}$ e fotoperíodo de 12 horas. Iniciada a emergência o filme plástico foi retirado e as plântulas permaneceram na BOD por 22 dias. Após este período, foram transplantadas em vasos e mantidas em casa de vegetação.

A capacidade dos vasos era de $3 \mathrm{dm}^{3}$, contendo solo classificado como LATOSSOLO VERMELHO distrófico e álico (Embrapa, 1999) do município de Montes Claros, coletado na camada de 0 a $20 \mathrm{~cm}$ de profundidade com os seguintes atributos químicos e granulometria, conforme Embrapa (1997): pH em água 4,6; P = 0,6 mg dm${ }^{-3} ; \mathrm{Ca}=1,1 \mathrm{mmol}$ $\mathrm{dm}^{-3} ; \mathrm{Mg}=0,4 \mathrm{mmol}_{\mathrm{c}} \mathrm{dm}^{-3} ; \mathrm{K}=0,01$ mmol $\mathrm{dm}^{-3} ; \mathrm{Al}=37 \mathrm{mmol}_{\mathrm{c}} \mathrm{dm}^{-3} ; \mathrm{H}+\mathrm{Al}$ $=120 \mathrm{mmol}_{\mathrm{c}} \mathrm{dm}^{-3}$, Matéria orgânica $=$ $24 \mathrm{~g} \mathrm{~kg}^{-1} ;$ areia $=500 \mathrm{~g} \mathrm{~kg}^{-1}$, silte $=80 \mathrm{~g}^{2}$ $\mathrm{kg}^{-1}$ e argila $=420 \mathrm{~g} \mathrm{~kg}^{-1}$. Em ensaios anteriores, aos 180 dias após a emergência das plantas, verificou-se que não houve influencia do tamanho do recipiente na qualidade das mudas, quando variaram de um a cinco litros de capacidade, justificando a utilização de vasos de três litros no presente estudo.
O delineamento experimental inteiramente casualizado, consta de 12 tratamentos, 4 repetições e duas plantas por vaso. Os tratamentos constaram da adubação com elemento faltante da seguinte forma: Completo 1 (C1-adubado com $\mathrm{N}, \mathrm{P}, \mathrm{K}, \mathrm{S}, \mathrm{B}, \mathrm{Cu}, \mathrm{Fe}, \mathrm{Zn}$ e calagem), completo 2 (C2- adubado com N, P, K, $\mathrm{S}, \mathrm{Ca}, \mathrm{Mg}, \mathrm{B}, \mathrm{Cu}, \mathrm{Fe}, \mathrm{Zn}$, sem calagem), tratamentos completos omitindo-se quando pertinente cada um dos nutrientes (completo $1-\mathrm{N}$, completo $1-\mathrm{K}$, completo 1 -S, completo 1 -P, completo 1 $\mathrm{B}$, completo $1-\mathrm{Zn}$, completo $2-\mathrm{Ca}$, completo $2 \mathrm{-Mg}$ ), testemunha (solo ao natural) e testemunha mais calagem. Para os tratamentos que receberam calcário, as doses foram baseadas em curvas de incubação determinadas via experimentos de laboratório, adotandose o critério de aumentar a saturação por bases (V) a 70\%. O corretivo foi o calcário dolomítico calcinado, micro pulverizado, com $36 \%$ de $\mathrm{CaO}, 14 \%$ de $\mathrm{MgO}$ (PRTN igual a $100 \%), 0,02 \%$ de $\mathrm{Zn}, 0,01 \%$ de $\mathrm{B}, 0,03 \%$ de $\mathrm{Fe}, 0,03 \%$ de $\mathrm{Mg}$ e $0,00 \%$ de $\mathrm{Cu}$.

As doses das fontes foram calculadas atendendo a adubação básica de cada tratamento, de acordo com Malavolta (1980): 100 mg de N, 300 mg de P, 100 $\mathrm{mg}$ de $\mathrm{K}, 200 \mathrm{mg}$ de $\mathrm{Ca}, 60 \mathrm{mg}$ de $\mathrm{Mg}$, $40 \mathrm{mg}$ de $\mathrm{S}, 0,5 \mathrm{mg}$ de $\mathrm{B}, 1,5 \mathrm{mg}$ de $\mathrm{Cu}$, $0,5 \mathrm{mg}$ de $\mathrm{Zn}$. Foram utilizados como fontes os seguintes sais p.a: $\mathrm{NH}_{4} \mathrm{NO}_{3}$, $\mathrm{H}_{3} \mathrm{PO}_{4}, \mathrm{KH}_{2} \mathrm{PO}_{4}, \mathrm{MgSO}_{4}, \mathrm{CaSO}_{4} .2 \mathrm{H}_{2} \mathrm{O}$, $\mathrm{Na}_{2} \mathrm{SO}_{4}, \mathrm{CuSO}_{4} .5 \mathrm{H}_{2} \mathrm{O}, \mathrm{H}_{3} \mathrm{BO}_{3}, \mathrm{ZnCl}_{2}$. No tratamento $\mathrm{C} 2$, o Ca e o $\mathrm{Mg}$ foram fornecidos na forma de $\mathrm{CaSO}_{4} \cdot 2 \mathrm{H}_{2} \mathrm{O}$ e $\mathrm{MgSO}_{4} \cdot 7 \mathrm{H}_{2} \mathrm{O}$, respectivamente.

Aos 135 dias após o transplantio das mudas, foram medidas a altura $(\mathrm{cm})$ e o diâmetro (mm) do colo de cada planta, separando-se parte aérea e raiz. Determinou-se a massa seca (g) da parte aérea (caule + folhas) e das raízes, teor de nutrientes na parte aérea e o teor de flavonóides totais nas folhas. Em ensaios preliminares verificou-se a correlação positiva entre teores de flavonóides totais das folhas e das vagens de favad'anta $\left(\mathrm{R}=0,76^{* *}\right)$. O teor de flavonóides totais foi determinado segundo metodologia proposta por Mendes et al. (2005).

Todas as variáveis foram submetidas à análise de variância e os tratamen- tos foram comparados pelo teste Scott Knott a $1 \%$ de probabilidade.

\section{RESULTADOS E DISCUSSÃO}

Como indicadores do crescimento, utilizaram-se os dados de produção de massa seca da raiz e da parte aérea, altura e o diâmetro do colo (Figura 1).

Nos tratamentos com omissão da calagem a massa da raiz e parte aérea secas foram maiores, em relação ao tratamento completo 1 (Figura 1). Foi comprovada a capacidade da fava d'anta em tolerar a acidez do solo e os altos teores de Al trocável, revelando o potencial de uso na recuperação de áreas degradadas, como sugerido por Lorenzi (2002). Além disso, os resultados indicam a presença de eficiente mecanismo de absorção de nutrientes, haja visto, que em solos ácidos a maioria dos elementos essenciais ao aparato fisiológico das plantas estão indisponíveis (Marschner, 1995).

$\mathrm{Na}$ ausência de calagem (C2), de calagem e $\mathrm{Ca}(\mathrm{C} 2-\mathrm{Ca})$ e calagem e $\mathrm{Mg}$ (C2 -Mg), tanto a massa seca da parte aérea quanto à de raiz, foram maiores que o tratamento completo (C1) (Figura 1). A adaptação da fava-d'anta às condições do cerrado (terrenos carentes em nutrientes e ácidos) justifica a ausência de resposta à calagem e a omissão de $\mathrm{Ca} \mathrm{e} \mathrm{Mg}$. Oliveira Júnior et al. (2004) também não verificaram diferenças na biomassa fresca e seca total de plantas de arnica (Lychnophora pinaster) cultivadas sem e com calagem. Segundo Rosolem (1989), as raízes das leguminosas têm capacidade de troca de cátions (CTC radicular) maior, com potencial de eficiência na absorção de Ca e Mg. O conteúdo do $\mathrm{Mg}$ na semente de algumas espécies vegetais pode assegurar o crescimento inicial das plantas sem prejuízo nutricional às mesmas (Malavolta, 1980).

Em plantas de jatobá, Duboc et al. (1996) relataram que na omissão de magnésio a resposta foi satisfatória em relação ao crescimento, com indiferença ao $\mathrm{Mg}$ faltante. Segundo Carniel et al. (1993), a embaúba (Cecropia sp.), o angico amarelo (Peltophorum dubium) e o fedegoso (Senna macranthera), durante o crescimento inicial, a altura, o diâmetro e a massa seca são variáveis indiferentes à omissão de magnésio. 


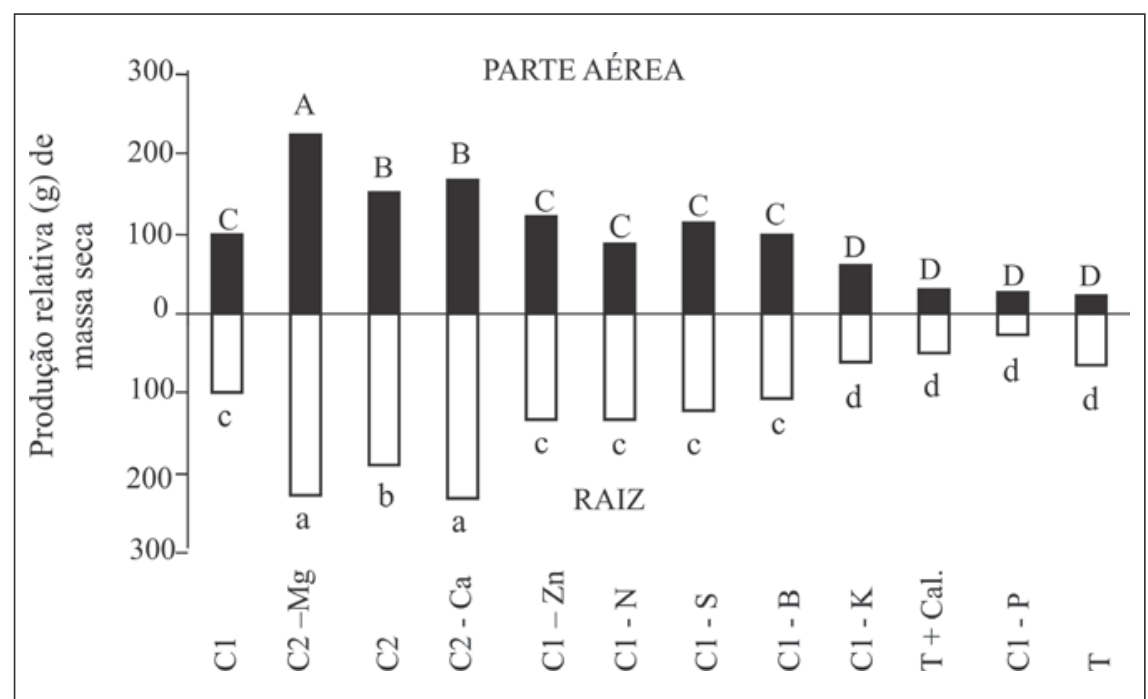

Figura 1. Crescimento relativo de plantas de fava-d'anta em comparação ao tratamento completo $1(\mathrm{C} 1)$. As médias seguidas pela mesma letra, maiúscula na parte aérea e minúscula na raiz, não diferem entre si pelo teste de Scott Knott a 1\% de probabilidade (Relative growth of fava-d'anta plants, in comparison to the complete treatment 1 (C1). Means followed by the same letter, large in the aerial part and small in the root, did not differ from each other by the Scott Knott test, $\mathrm{P}<0.01$ ). Montes Claros, UFMG, 2005.

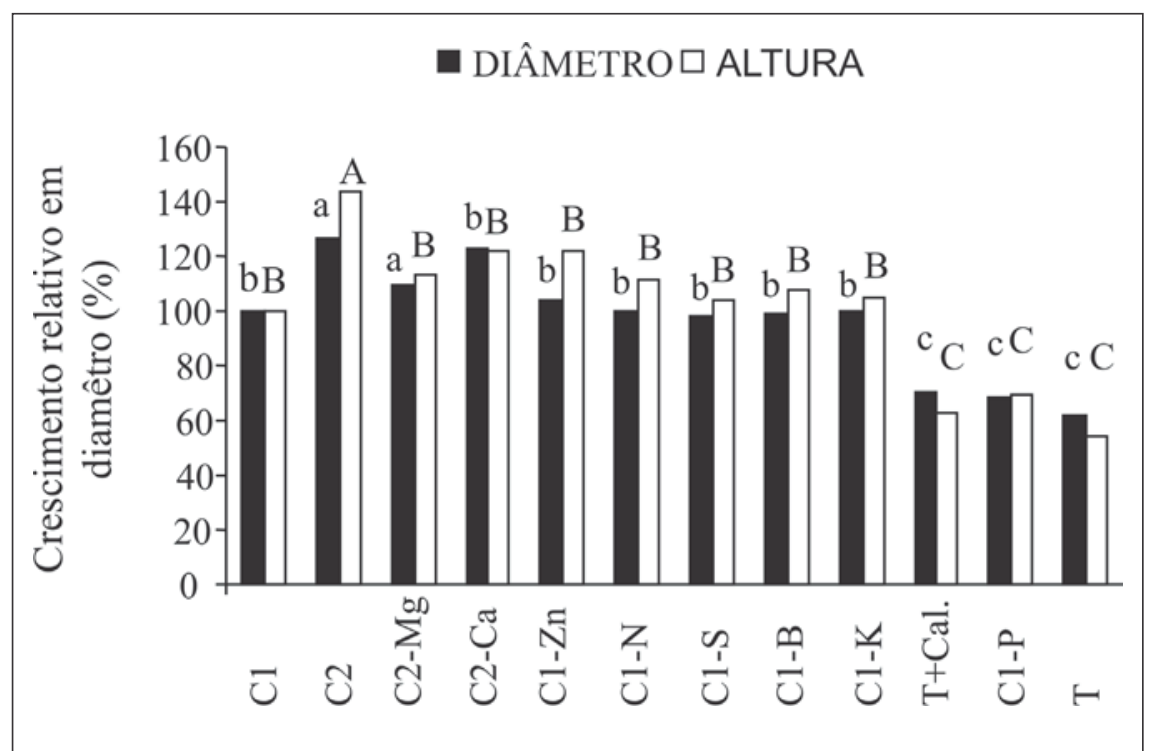

Figura 2. Crescimento relativo de plantas de fava-d'anta em função da altura e diâmetro do colo e ao tratamento Completo 1 (C1). As médias seguidas pela mesma letra, maiúscula altura e minúscula diâmetro do colo, não diferem entre si pelo teste de Scott Knott a $1 \%$ de probabilidade (Relative growth of fava-d'anta plants as a function of height and diameter of the fava d'anta and the complete treatment $1(\mathrm{C} 1)$. Means followed by the same letter, large in the aerial part and small in the root, did not differ from each other by the Scott Knott test, $\mathrm{P}<0.01$ ). Montes Claros, UFMG, 2005.

A tolerância da planta à alta acidez do solo pode ser comprovada pelos tratamentos testemunha mais calagem e testemunha (solo ao natural). No primeiro caso pode-se afirmar que devido à elevação do $\mathrm{pH}$ a planta não cresceu, porém, quando omitiu-se a calagem (tra- fotoassimilados e condições do solo como textura, estrutura, umidade, $\mathrm{pH}$ e disponibilidade de nutrientes.

Algumas plantas apresentam mecanismos de adaptação em ambientes estressantes, como a baixa disponibilidade de nutrientes, de modo que suas funções fisiológicas não sejam afetadas (Epstein \& Bloom, 2006). Dessa forma, a fava-d'anta, em virtude da alta acidez do solo, não teve o crescimento radicular afetado quando omitiu-se cálcio (C2-Ca) e magnésio (C2-MG) e cálcio, quando omitiu-se o cálcio (C2-Ca) (Figura 1).

$\mathrm{O}$ aumento da saturação por bases a $70 \%$ pode ter prejudicado as plantas. Há necessidade de se conhecer na fava d'anta o valor ideal de saturação de bases.

Apesar de o $\mathrm{N}$ ser o nutriente que mais limita a produção, para leguminosa fava-d'anta, as massas secas da parte aérea e da raiz foram semelhantes ao tratamento com adubação completa (Figura 1). Embora não tenha sido objetivo do trabalho, verificou-se a presença de nódulos nas raízes das plantas no tratamento com omissão de nitrogênio.

Quanto ao Zn, B e S, a massa seca da parte aérea e raiz foi favorecida pelo tratamento sem calagem (C2) em relação ao $\mathrm{C} 1$. No caso do Zn, provavelmente, houve inibição competitiva com o Ca e Mg (Couto et al. 1985).

Os nutrientes que mais limitaram a massa seca da parte aérea e raiz foram $\mathrm{P}$ e K. O P está relacionado com compostos importantes das células vegetais, incluindo fosfato, açúcares intermediários da respiração e fotossíntese, bem como, os fosfolipídeos que compõem as membranas vegetais. Estando o $\mathrm{P}$ ausente de acordo com Taiz \& Zeiger (2004), as plantas cresceram menos formando caules delgados com senescência das folhas mais velhas, comprovando os sintomas visuais das plantas no tratamento com omissão de $\mathrm{P}$, deste estudo. Desta maneira, o P combinado a outros nutrientes é essencial na síntese de proteínas e na ativação de enzimas responsáveis pelo aumento da biomassa vegetal (Marschner, 1995).

Segundo Malavolta (1985), o P tem função fundamental na vida das plantas, participando dos compostos ricos em energia, como o trifosfato de adenosina (ATP), sendo absorvido pelas raízes como $\mathrm{H}_{2} \mathrm{PO}_{4}^{-}$. $\mathrm{O}$ aumento do 
Tabela 1. Massa seca da parte aérea e quantidade acumulada de macro e micronutrientes pelas plantas de fava-d'anta (Dimorphandra mollis) (Dry weight of aerial part and quantity of accumulated macro and micronutrients of fava-d'anta plants). Montes Claros, UFMG, 2005.

\begin{tabular}{|c|c|c|c|c|c|c|c|c|c|c|c|c|}
\hline \multirow{3}{*}{ Trat. } & \multirow{3}{*}{$\begin{array}{l}\text { Massa seca } \\
\text { parte aérea }(g)\end{array}$} & \multicolumn{11}{|c|}{ Quantidade acumulada } \\
\hline & & \multicolumn{6}{|c|}{$\mathrm{mg} / \mathrm{g}$} & \multicolumn{5}{|c|}{$\mathrm{mg} / \mathrm{g}$} \\
\hline & & $\mathbf{N}$ & $\mathbf{P}$ & $\mathbf{K}$ & $\mathrm{Ca}$ & $\mathrm{Mg}$ & $\mathbf{S}$ & $\mathbf{B}$ & $\mathrm{Cu}$ & Mn & $\mathrm{Zn}$ & $\mathrm{Fe}$ \\
\hline $\mathrm{C} 1$ & $4,58(100,0)^{\star}$ & 20,11 & 0,60 & 3,85 & 5,59 & 0,87 & 0,92 & 289,91 & 17,40 & 143,81 & 122,74 & 2181,91 \\
\hline $\mathrm{C} 2$ & $10,31(225,1)^{\star}$ & 38,15 & 1,34 & 7,11 & 0,82 & 1,24 & 1,44 & 481,48 & 57,74 & 489,73 & 361,88 & 1585,68 \\
\hline $\mathrm{C} 2-\mathrm{Ca}$ & $7,71(168,3)^{*}$ & 35,31 & 1,16 & 6,86 & 0,93 & 1,00 & 1,46 & 385,50 & 33,92 & 427,91 & 284,50 & 1318,41 \\
\hline $\mathrm{C} 2-\mathrm{Mg}$ & $7,00(152,8)^{\star}$ & 26,67 & 0,98 & 5,39 & 0,84 & 0,49 & 0,84 & 399,70 & 35,00 & 323,40 & 279,30 & 1586,20 \\
\hline $\mathrm{C} 1-\mathrm{Zn}$ & $5,62(122,7)^{*}$ & 22,31 & 0,67 & 4,83 & 7,64 & 1,41 & 0,96 & 331,02 & 17,98 & 165,23 & 60,13 & 2495,28 \\
\hline $\mathrm{C} 1-\mathrm{N}$ & $4,07(88,9)^{\star}$ & 15,83 & 0,49 & 2,40 & 3,74 & 0,73 & 1,10 & 268,21 & 16,28 & 191,70 & 111,93 & 1018,31 \\
\hline C1-S & $5,17(112,9)^{*}$ & 21,40 & 0,57 & 3,83 & 7,13 & 0,88 & 0,93 & 349,49 & 22,75 & 170,09 & 132,35 & 2456,27 \\
\hline C1-B & $4,44(96,9)^{*}$ & 19,62 & 0,53 & 3,20 & 4,00 & 0,71 & 0,80 & 103,01 & 22,20 & 165,17 & 120,77 & 3151,51 \\
\hline $\mathrm{C} 1-\mathrm{K}$ & $2,84(62,0)^{*}$ & 11,87 & 0,37 & 1,36 & 3,66 & 0,65 & 0,74 & 230,61 & 9,09 & 119,28 & 77,82 & 735,56 \\
\hline C1-P & $1,47(25,4)^{\star}$ & 6,48 & 0,09 & 1,18 & 1,03 & 0,29 & 0,22 & 92,02 & 7,79 & 49,10 & 31,61 & 1918,06 \\
\hline $\mathrm{T}+$ Cal. & $1,35(29,5)^{*}$ & 5,71 & 0,07 & 0,46 & 0,45 & 0,20 & 0,20 & 47,52 & 8,37 & 49,55 & 18,90 & 1467,05 \\
\hline $\mathrm{T}$ & $1,12(24,4)^{*}$ & 3,96 & 0,06 & 0,52 & 0,09 & 0,07 & 0,16 & 49,84 & 6,72 & 62,05 & 18,59 & 764,51 \\
\hline
\end{tabular}

*Produção relativa, em percentagem, da massa seca de cada tratamento em relação ao tratamento Completo 1 (C1) (Relative yield, in percent, of the dry matter of each treatment in relation to the complete treatment $1(\mathrm{C} 1))$.

Tabela 2. Teor de flavonóides totais em folhas de fava d'anta (Dimorphandra mollis) nos diferentes tratamentos (Total flavonoids content in leaves of fava-d'anta plants comparing different treatments). Montes Claros, UFMG, 2005.

\begin{tabular}{ccccccccccccc}
\hline \multicolumn{11}{c}{ Teor de flavonóides totais (\%) } \\
\hline $\mathrm{C} 1^{*}$ & $\mathrm{C} 1-\mathrm{N}$ & $\mathrm{C} 1-\mathrm{K}$ & $\mathrm{C} 1-\mathrm{S}$ & $\mathrm{C} 1-\mathrm{P}$ & $\mathrm{C} 1-\mathrm{B}$ & $\mathrm{C} 1-\mathrm{Zn}$ & $\mathrm{C} 2$ & $\mathrm{C} 2-\mathrm{Ca}$ & $\mathrm{C} 2-\mathrm{Mg}$ & $\mathrm{T}$ & $\mathrm{T}-\mathrm{Cal}$. & Média \\
0,27 & 0,24 & 0,22 & 0,25 & 0,21 & 0,25 & 0,24 & 0,30 & 0,27 & 0,23 & 0,26 & 0,30 & 0,25 \\
\hline
\end{tabular}

* Completos 1 (C1), C1 sem N (C1-N), C1 sem K (C1-K), C1 sem S (C1-S), C1 sem P (C1-P), C1 sem B (C1-B), C1 sem Zn (C1-Zn), Completo 2 (C2), C2 sem Ca (C2-Ca), C2 sem Mg (C2-Mg), Testemunha (T) e Testemunha mais calagem (T+Cal.) (Complete 1 (C1), C1 without $\mathrm{N}(\mathrm{C} 1-\mathrm{N}), \mathrm{C} 1$ without $\mathrm{K}(\mathrm{C} 1-\mathrm{K}), \mathrm{C} 1$ without $\mathrm{S}(\mathrm{C} 1-\mathrm{S}), \mathrm{C} 1$ without $\mathrm{P}(\mathrm{C} 1-\mathrm{P}), \mathrm{C} 1$ without B (C1-B), C1 without Zn (C1-Zn), Complete $2(\mathrm{C} 2), \mathrm{C} 2$ without $\mathrm{Ca}(\mathrm{C} 2-\mathrm{Ca}), \mathrm{C} 2$ without $\mathrm{Mg}(\mathrm{C} 2-\mathrm{Mg})$, Check treatment $(\mathrm{T})$ and $\mathrm{Check}$ treatment $+\mathrm{Ca}(\mathrm{T}+\mathrm{Ca}))$.

$\mathrm{pH}$ incrementa a disponibilidade dos fosfatos no solo. Porém, não são aplicáveis à fava-d'anta, já que esta planta produziu maior massa seca da parte aérea e raiz quando o $\mathrm{pH}$ não respondeu a adição de calcário.

Pode-se inferir que a forma de absorção de fontes fosfatadas pela fava d'anta pode não ser a forma mais comum que consta da bibliografia consultada, em geral $\left(\mathrm{H}_{2} \mathrm{PO}_{4}^{-}\right)$e sim formas menos disponíveis como os fosfatos de Fe e Al típicos dos solos do Cerrado.

Na ausência de K a massa seca da parte aérea e raiz foi significativamente menor que no tratamento $\mathrm{C} 1$. O potássio desempenha importante função de regulação do potencial osmótico das células vegetais, além de ativar enzimas da respiração e fotossíntese (Taiz \& Zeiger, 2004). Na presença de K, mesmo sem calagem, a massa seca da parte aérea e raiz foi semelhante ao $\mathrm{C} 1$. Em plantas de nogueira (Carya $\mathrm{sp}$ ) cultivadas em solução nutritiva, a absorção de
$\mathrm{K}$ foi independente do aumento de concentração de Al (Zysset et al., 1996).

A altura e diâmetro da fava d'anta maiores no tratamento completo 2 (C2) (Figura 2) e menores nos tratamentos completo 1 sem P (C 1 -P), testemunha (solo ao natural) e testemunha mais calagem (T+Cal.) (Figura 2). Dessa forma, apesar de ser tolerante a níveis altos de acidez do solo, a fava d'anta necessita de nutrientes, principalmente $\mathrm{P}$, para o crescimento em altura e diâmetro do colo.

O diâmetro é considerado a variável mais eficiente para indicar o padrão de qualidade das mudas, tendo alta correlação com a porcentagem de sobrevivência após transplante (Sturion, 1981).

Maiores quantidades de nutrientes ocorreram no tratamento $\mathrm{C} 2$, exceto quanto ao cálcio, que teve seu maior acúmulo no tratamento $\mathrm{C} 1$ (Tabela 1). $\mathrm{O}$ maior acúmulo de nutrientes no tratamento C2 decorreu da maior massa seca da parte aérea em relação aos demais.
De modo geral, em ordem decrescente, os nutrientes acumulados em maiores quantidades foram: $\mathrm{N}>\mathrm{K}>\mathrm{Ca}$ $>\mathrm{S}>\mathrm{Mg}>\mathrm{Fe}>\mathrm{B}>\mathrm{Mn}>\mathrm{Zn}>\mathrm{Cu}$ (Tabela 1).

O teor de flavonóides totais nas folhas não variou significativamente entre os tratamentos, sendo o teor médio 0,25\% (Tabela 2). Segundo Correa Jr. et al. (1991), a fertilização de plantas medicinais dificilmente altera a produção de fitofármacos podendo contribuir no aumento da biomassa. Em camomila (Chamomilla recutita), Wagner \& Bladt (1996) observaram que o teor de óleo essencial dos capítulos florais não variou com adubação orgânica e espaçamentos. Também em camomila, Mapeli et al. (2005), não observaram diferença quanto ao teor de óleos essenciais nas plantas submetidas a doses de $\mathrm{N}$ e P. Estes tratamentos foram eficientes apenas em aumentar a altura, a biomassa total das plantas e o número de capítulos florais. 
Embora não se tenha observado efeito dos tratamentos no teor de flavonóides totais, a produção de massa seca da parte aérea foi significativamente influenciada pelos tratamentos (Tabela 2), podendo-se inferir que nos tratamentos que causaram maior crescimento das plantas, a quantidade de flavonóides totais também foi maior.

A fava d'anta mostrou-se tolerante à acidez e sensível à elevação da saturação por bases do solo a $70 \%$ e a omissão de P e K. Por outro lado, os teores de flavonóides totais não foram influenciados pelos tratamentos estudados.

\section{AGRADECIMENTOS}

À FAPEMIG pelo apoio.

\section{REFERÊNCIAS}

ALMEIDA SP. 1998. Cerrado: espécies vegetais úteis. Planaltina: Embrapa. 464p.

BRAGA FA; VALE FR; VENTORIM N; AUBERT E; LOPES GA. 1995. Requerimentos nutricionais de quatro espécies florestais. Revista Árvore 19: 18-31.

CARNIEL T; LIMA HN, VALE FR; SIQUEIRA JO; CURI N; GOMES RJ. 1993. Resposta à adubação no campo de cinco espécies arbóreas nativas do sudeste brasileiro. In: CONGRESSO BRASILEIRO DE CIÊNCIA DO SOLO, 1993, Goiânia . Resumos... Goiânia: SBCS. p.209-210.

CORREA JUNIOR C. 1991. Cultivo de plantas medicinais, codimentares e aromáticas. Curitiba: Emater - Paraná. 151p.

COUTO C; NOVAIS RF; BARROS NF; NEVES JCL. 1985. Resposta do eucalipto à aplicação de zinco em amostras de solos de cerrado. Revista Árvore 9: 134-148.

DECHEN AR; FURLANI AMC; FURLAN PR 1996. Tolerância e adaptação de plantas aos estresses nutricionais. In: SIQUEIRA JO; MOREIRA FMS; LOPES AS; GUILHERME LRG; FAQUIN V; FURTINI NETO AE; CARVALHO JG. (eds.). Inter-relação fertilidade biologia do solo e nutrição de plantas. Viçosa: SBCS; Lavras: UFLA/DCS. p.337-361.

DUBOC E; VENTORIM N; VALE FR; DAVIDE AC. 1996. Nutrição do jatobá. Revista Ciência e Agrotecnologia 2: 138-152.
EMPRESA BRASILEIRA DE PESQUISA AGROPECUÁRIA - Centro Nacional de Pesquisa da Amazônia Oriental, (EMBRAPACPATU. Documentos), 1997, p.148.

EMPRESA BRASILEIRA DE PESQUISA AGROPECUÁRIA - Centro Nacional de Pesquisa de Solos. 1999. Sistema Brasileiro de Classificação de Solos. Brasília: Embrapa Produção de Informação; Rio de Janeiro: Embrapa Solos. 412p.

EPSTEIN E; BLOOM AJ. 2006. Nutrição mineral de plantas: princípios e perspectivas. 2 ed. Londrina: Editora Planta. 403p.

FERREIRA RA; BOTELHO SA; DAVIDE AC; MALAVASE MM. 2001. Morfologia de frutos, sementes, plântulas e plantas jovens de Dimorphandra mollis Benth. - faveira (Leguminosae-Caesalpinioideae). Revista Brasileira de Botânica 24: 303-309.

LORENZI H. 2002. Árvores brasileiras: manual de identificação e cultivo de plantas arbóreas nativas do Brasil. v.2. 4 ed.Nova Odessa: Editora Plantarum. p. 368.

MALAVOLTA E. 1980. Elementos de nutrição de plantas. São Paulo: Agronômica Ceres. 251p.

MALAVOLTA E. 1985. Nutrição mineral. In: FERRI MG. (Coord.). Fisiologia vegetal. 2 ed. São Paulo: EPU. p.97-114.

MAPELI NC; VIEIRA MC; HEREDIA ZNA; SIQUEIRA JM. 2005. Produção de biomassa e de óleo essencial dos capítulos florais de camomila em função de nitrogênio e fósforo. Horticultura Brasileira 23: 32-37.

MARSCHNER H. 1995. Mineral nutrition of higher plants. 2 ed. New York: Academic Press. p. 889-901.

MATOS RMB; SILVA EMR; LIMAE. 1999. Fungos micorrízicos e nutrição de plantas. Seropédica: Embrapa Agrobiologia. 36 p.

MELO JT; LIMA VLGF; RIBEIRO JF. 1981. Desenvolvimento inicial de Astronium urundeuva Fr. All. Engl. (aroeira) em diferentes tipos de solo da região dos Cerrados. In: CONGRESSO NACIONAL DE BOTÂNICA, 32., 1981, Teresina. Anais... Teresina: SBB. p. 283-298.

MENDES ADR; MARTINS ER; FERNANDES, LA; MARQUES CCL. 2005. Produção de biomassa e de flavonóides totais por fava d'anta (Dimorphandra mollis Benth) sob diferentes níveis de fósforo em solução nutritiva. Revista Brasileira de Plantas Medicinais 7: 7-11.
OLIVEIRA JÚNIOR AC; FANQUIN V; PINTO JEBP; SOBRINHO RRL; BERTOLUCCI SKV; TEIXEIRANR. 2004. Teor e rendimento de óleo essencial de mudas de arnica, em função de calagem e adubação. Horticultura Brasileira v. 22, n. 2. Suplemento 2. CD-ROM. Trabalho apresentado no $44^{\circ}$ Congresso Brasileiro de Olericultura, 2004.

PANEGASSI VR; SERRA GE; BUCKERIDGE MS. 2000. Potencial tecnológico do galactomanano de sementes de faveiro (Dimorphandra mollis) para uso na indústria de alimentos. Ciência e Tecnologia de Alimentos 20: 1-28.

PERES LEP; KERBAUY G B. 2000. Controle hormonal do desenvolvimento das raízes. $R e$ vista Universa 8: 181-195.

RENÓ NB; VALE FR; CURI N; SIQUEIRA JO. 1993. Requerimentos nutricionais de quatro espécies florestais nativas. In: CONGRESSO BRASILEIRO DE CIÊNCIA DO SOLO 24, 1993, Goiânia. Resumos... Goiânia: SBCS. p.211-212.

RIBEIRO AQ; LEITE JP; DANTAS-BARROS AM. 2005. Perfil de utilização de fitoterápicos em farmácias comunitárias de Belo Horizonte sob a influência da legislação nacional. Revista Brasileira de Farmacognosia 15: 65-70.

ROSOLEM CA. 1989. Interpretação dos teores de bases trocáveis do solo. In: BÜLL LT; ROSOLEM CA. (Eds.). Interpretação de análise química de solo e planta para fins de adubação. Botucatu: Fundação de Estudos e Pesquisas Agrícolas e Florestais. p.97-128.

SANTOS MD; BLATT CTT. 1998. Teor de flavonóides e fenóis totais em folhas de Pyrostegia venusta Miers. de mata e de cerrado. Revista Brasileira de Botânica 21: 1-9.

STURION JA. 1981. Produção de mudas Mimosa scabella Benth. In: SEMINÁRIO SOBRE ATUALIDADES E PERSPECTIVAS FLORESTAIS. "BRACATINGA UMA ALTERNATIVA PARA REFLORESTAMENTO”, 4., 1981, Curitiba. Anais... Curitiba: EMBRAPA/ URPFCS. p.19-35.

TAIZ L; ZEIGER E. 2004. Fisiologia vegetal. Califórnia: Artmed. 719p.

WAGNER H; BLADT S. 1996. Plant drug analysis - a thin layer chromatography atlas. 2.ed. Berlin: Springer. 384 p.

ZYSSET M; BRUNNER I; FREY B; BLASER P. 1996. Response of european chestnut to varying calcium/aluminum ratios. Journal of Environmental Quality 25: 702-708. 